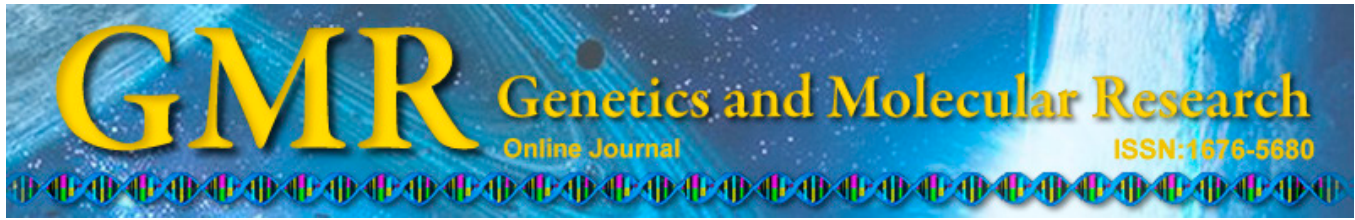

\title{
Verification of specific selection SNPs between broiler and layer chicken in Chinese indigenous chicken breeds
}

\author{
D. Lan*, Y.D. Hu*, Q. Zhu, D.Y. Li and Y.P. Liu
}

Farm Animal Genetic Resources Exploration and Innovation Key Laboratory of Sichuan Province, Sichuan Agricultural University, Ya'an, Sichuan, China

*These authors contributed equally to this study.

Corresponding author: Y.P. Liu

E-mail: 525692787@qq.com

Genet. Mol. Res. 14 (3): 8388-8396 (2015)

Received November 4, 2014

Accepted April 24, 2015

Published July 28, 2015

DOI http://dx.doi.org/10.4238/2015.July.28.5

\begin{abstract}
The direction of production for indigenous chicken breeds is currently unknown and this knowledge, combined with the development of chicken genome-wide association studies, led us to investigate differences in specific loci between broiler and layer chicken using bioinformatic methods. In addition, we analyzed the distribution of these seven identified loci in four Chinese indigenous chicken breeds, Caoke chicken, Jiuyuan chicken, Sichuan mountain chicken, and Tibetan chicken, using DNA direct sequencing methods, and analyzed the data using bioinformatic methods. Based on the results, we suggest that Caoke chicken could be developed for meat production, while Jiuyuan chicken could be developed for egg production. As Sichuan mountain chicken and Tibetan chicken exhibited large polymorphisms, these breeds could be improved by changing their living environment.
\end{abstract}

Key words: Molecular marker; Polymorphic site; Indigenous breeds; Breeding direction 


\section{INTRODUCTION}

Specialized commercial populations, which originate in foreign countries, occupy the majority of the chicken market in china. Therefore, it is necessary to take full advantage of indigenous breeds that have large genetic resources and play an important role in maintaining biological polymorphism, as well as having enhanced production performance, such as being well-adapted, resistant to foraging, and stress tolerant, etc. Although, these indigenous may also have some disadvantages, such as low production performance. The first limiting factor for the development of indigenous breeds is the unknown direction of production, which is the first and foremost requirement when studying indigenous breeds.

Reproduction and growth traits are controlled by quantitative trait loci and a genomewide scan is a powerful approach that can be used to gain an understanding of these complex traits. Recently, many investigations have used genome-wide association studies (GWAS) to identify special loci used during chicken domestication (van Kaam et al., 1999; Useche et al., 2001; Akey et al., 2002; Reich et al., 2003; Rubin et al., 2010; Gu et al., 2011; Xie et al., 2012; Li et al., 2012, 2013). Rubin et al. (2010) used whole-genome re-sequencing methods to analyze chicken selection loci and the results revealed that there are 7,000,000 single nucleotide polymorphisms (SNPs), including 1300 deficiency loci, and during chicken domestication specific loci are involved in specialization into broiler (meat-producing) and layer (eggproducing) chicken. A follow-up study indicated that the high-growth and low-growth lines of broiler were distinguished by an 18,961-bp deficiency. Using a GWAS, Gu et al. (2011), found that the chicken chromosome 4 (GGA4) region, approximately $8.6 \mathrm{Mb}$ in length (71.6 to $80.2 \mathrm{Mb}$ ), had a large number of significant SNP effects for late growth during weeks 7 to 12. Furthermore, Xie et al. (2012), using a GWAS, found a narrow 1.5 Mb region (173.5 to 175 $\mathrm{Mb}$ ) of chicken (Gallus gallus) chromosome (GGA) 1 to be strongly associated with chicken growth.

Previous research indicates that mutations developed during chicken domestication are relatively fixed for different traits. In this study, molecular biological techniques are used to explore the breeding direction of Sichuan indigenous breeds by detecting the specific loci in each breed.

\section{MATERIAL AND METHODS}

\section{Birds and management}

A total of 193 chickens belonging to four different Chinese indigenous breeds were sampled for this study: Tibetan chicken, Caoke chicken, Jiuyuan chicken, and Sichuan mountain chicken. All of these breeds are famous Chinese indigenous chicken breeds and are mainly distributed in Sichuan Province, China. The selected chickens were all from unimproved breeds. The distribution of sampled chickens and the number of samples collected are shown in Table 1.

All birds were reared under the same condition: cage free (density of $<35$ chicken $/ 100$ $\left.\mathrm{m}^{2}\right)$ and standard conditions of temperature $\left(20^{\circ}\right.$ to $\left.22^{\circ} \mathrm{C}\right)$, relative humidity $(55$ to $60 \%)$, and ventilation were maintained. The chickens were fed the same professional breeder diet and had free access to feed and water during the entire rearing period. Birds were managed with full consideration of bird welfare. All procedures involving animals were approved by the Institutional Animal Care and Use Committee of the National Institute of Agrobiological Sciences. 
Table 1. Distribution and number of experimental chicken sampled for each Chinese indigenous breed.

\begin{tabular}{lcl}
\hline Breed & No. of samples & Distribution \\
\hline Tibetan chicken (TC) & 42 & Xiangcheng Garze \\
Caoke chicken (CK) & 54 & Simian County, Sichuan \\
Jiuyuan black chicken (JY) & 48 & Wangyuan County, Sichuan \\
Sichuan mountain chicken (SM) & 49 & Junlian County, Sichuan \\
\hline
\end{tabular}

\section{Sample collection}

Approximately $2 \mathrm{~mL}$ blood was collected from the brachial vein of each individual according to the requirements of animal welfare of Sichuan Agricultural University, and stored at $-20^{\circ} \mathrm{C}$. The next day, genomic DNA was isolated by phenolic extraction. The quality and integrity of DNA was assessed using the $\mathrm{A}_{260 / 280}$ ratio and agarose gel electrophoresis.

\section{Objective gene screening}

Firstly, we undertook a literature review to determine the genes related to animal reproduction traits, and then we identified the genes on the local chromosome using NCBI (http://www.ncbi.nlm.nih.gov/). Secondly, we determined the variable loci within the genes, the amount and type of mutation in specialized layer and broiler chicken using chicken VD data (http://chicken.genomics.org.cn/), and then assessed if there were differences in the loci between specialized layer and broiler chicken. Thirdly, the primers for the candidate differential gene loci were designed and verified on specialized layer and broiler chickens to exclude primers with a false position. Finally, we selected loci on three genes as the objective gene loci to detect genetic variation in the four Chinese indigenous chicken breeds.

\section{Analysis of genetic variation}

Following confirmation of the objective genes, the primers were designed using Primer Primer 5.0, DNA sequence fragments were amplified, and genetic variation was analyzed using the direct sequencing method by Beijing Liuhe Genomics Biological Technology Co., Ltd. (Beijing, China).

\section{Sequence and data analysis}

Chromas version 1.45 was used to obtain the original sequence data and DNA Star was used to inspect, edit, and proof the sequences to ensure data accuracy. To determine whether the sequences obtained were correct, an NCBI nucleotide BLAST search was conducted (http://blast.ncbi.nlm.nih.gov/). MEGA4.1 and DnaSP were used to analyze variation in the loci and identify haplotypes.

The genetic variation within each indigenous breed was evaluated and compared. The total number of alleles at each locus, the respective allele frequency, observed and expected $\left(H_{\mathrm{E}}\right)$ heterozygosities, and polymorphism information content (PIC) value for each breed across the loci were calculated using GenAlEx 6.4 (Peakall and Smouse, 2012) and CERVUS ver. 3.0.3 (Kalinowski et al., 2007). 


\section{RESULTS}

\section{Genetic screening and primer design}

The study identified 7 SNPs on three genes as the differential genes between specialized layer and broiler chicken. The 7 SNPs included three loci specific to layer chicken, chr24:5748784A $>\mathrm{G}$, chr24:5748832T $>\mathrm{C}$, and chr5:52922938A $>\mathrm{G}$, and four loci specific to broiler chicken, chr3:7519896A $>$ G, chr3:7519918G $>$ A, chr5:52926659T $>A$, and chr5:52926664T $>$ C. These 7 SNPs are located on the DRD2, LHCGR, and ESR2 genes, respectively (Table 2), all of which are reproductive trait-related genes. The primers were designed according to the gene loci (GenBank accession No. NC_006092.3, NC_006090.3, NC_006111.3), as shown in Table 2.

\begin{tabular}{|c|c|c|c|c|c|}
\hline Gene & SNP locus & Primer & Sequences of primer & $\begin{array}{l}\text { Amplification } \\
\text { fragment (bp) }\end{array}$ & $\begin{array}{c}\text { Annealing } \\
\text { temperature }\left({ }^{\circ} \mathrm{C}\right)\end{array}$ \\
\hline \multirow[t]{2}{*}{ ESR2 } & Chr5:52922914A $>G$ & 1 & $\begin{array}{l}\text { Forward: 5'-CCAGATAGTGGACCAAGCC-3' } \\
\text { Reverse: 5'-CCTCGTAAACAGCCATTCC-3' }\end{array}$ & 705 & 58.2 \\
\hline & $\begin{array}{l}\text { Chr5:52926659A }>\mathrm{T} \\
\text { Chr5:52926664C }>\mathrm{T}\end{array}$ & 2 & $\begin{array}{l}\text { Forward: 5'-GATTTGATGCCCGATGAG-3' } \\
\text { Reverse: 5'-CTGCTACACTGGGAAGACC-3' }\end{array}$ & 517 & 54.1 \\
\hline$L H C G R$ & $\begin{array}{l}\text { Chr3:7519896G }>A \\
\text { Chr3:7519918G }>\text { A }\end{array}$ & 3 & $\begin{array}{l}\text { Forward: 5'-CAAGAGGCAGATGTAAAGACT-3' } \\
\text { Reverse: 5'-TTAGACCCAGGATGTGAGAA-3' }\end{array}$ & 960 & 53.2 \\
\hline$D R D 2$ & $\begin{array}{l}\text { Chr24:5748832T }>C \\
\text { Chr24:5748784A }>\text { G }\end{array}$ & 4 & $\begin{array}{l}\text { Forward: 5'-ACATCGGCTATTTCCAGAC-3' } \\
\text { Reverse: 5'-CAACTGCTTGCTCTTCTCA-3' }\end{array}$ & 555 & 55.2 \\
\hline
\end{tabular}

\section{Genetic diversity analysis}

Table 3 shows the results of the genetic diversity analysis for each chicken breed. The Chr5:52922938A > T locus was homozygous and the $H_{\mathrm{E}}$ values were zero in the Sichuan mountain chicken breed, while this locus had low polymorphism $(\mathrm{PIC}<0.25)$ in the Caoke chicken breed, and moderate polymorphism $(0.25<$ PIC $<0.5)$ in the Tibetan chicken and Jiuyuan black chicken breeds. The Chr5:52926659T $>\mathrm{A}$ and $\mathrm{Chr} 5: 52926664 \mathrm{C}>\mathrm{T}$ loci exhibited moderate polymorphism in all of four chicken breeds, except for Chr5:52926664C $>\mathrm{T}$, which exhibited low polymorphism and reached Hardy-Weinberg equilibrium $(\mathrm{P}>0.05)$ in the Jiuyuan black chicken breed. Apart from the Jiuyuan black chicken breed, which has low polymorphism, the other three breeds all have moderate polymorphism at the Chr3:7319896G $>\mathrm{A}$ and Chr3:7519918G $>$ A loci. The Chr3:7319896G $>$ A locus was in Hardy-Weinberg equilibrium $(\mathrm{P}>0.05)$ in Sichuan mountain chicken, while the Chr3:7519918G $>$ A locus was in HardyWeinberg equilibrium $(\mathrm{P}>0.05)$ in three of the four breeds, except the Caoke chicken breed. The Chr24:5748832 T>C locus was homozygous in the Caoke chicken breed, while the other three breeds exhibited moderate polymorphism for this locus. All of the experimental breeds exhibited moderate polymorphism for the Chr24:5748784A $>\mathrm{G}$ locus.

\section{Genotype and allele analysis}

Table 4 shows the genotypes and allele frequencies for the four chicken breeds. The Chr5:52922938A $>$ G locus has three genotypes (GG, AA, and AG). Each genotype has a dif- 
ferent frequency of occurrence: Jiuyuan black chicken only have the AG genotype, Sichuan mountain chicken only have the GG genotype, Tibetan chicken have both the AA and GG genotypes, while Caoke chicken has all three genotypes. The Chr5:52922938A>T locus has three genotypes (AA, AT, and TT). The three genotypes occur with varying frequency in each of the four breeds (Table 4). The Chr5:52926664C $>\mathrm{T}$ locus is composed of three genotypes (CC, CT, and TT), which occur with varying frequency in the four chicken breeds, except the TT genotype, which is not present in Jiuyuan chicken (Table 4). The GG genotype of the Chr3:7319896G $>$ A locus is mainly observed in Caoke chicken and Jiuyuan black chicken, with probabilities of 52 and $88 \%$, respectively, while the AA genotype is mainly observed in Tibetan chicken and Sichuan mountain chicken, with probabilities of 74 and 55\%, respectively.

Table 3. Genetic diversity for each of the four Chinese indigenous chicken breeds for each of the seven SNP loci.

\begin{tabular}{|c|c|c|c|c|c|c|c|}
\hline SNP & Breeds & $H_{\mathrm{O}}$ & $H_{\mathrm{E}}$ & $N_{\mathrm{A}}$ & PIC & $\chi^{2}$ & $P$ \\
\hline \multicolumn{8}{|c|}{ Chr5: 52922938} \\
\hline \multirow[t]{5}{*}{$A>G$} & CK & 0.698819 & 0.301181 & 1.430986 & 0.134979 & 1.239669 & 0.265535 \\
\hline & JY & 0.5 & 0.5 & 2 & 0.375 & 48 & $<0.0001$ \\
\hline & SM & 1 & 0 & 1 & 0 & - & - \\
\hline & $\mathrm{TC}$ & 0.529744 & 0.470256 & 1.887704 & 0.359686 & 15.90533 & $<0.0001$ \\
\hline & Total & 0.502269 & 0.497731 & 1.99096 & 0.373863 & 1.540589 & 0.2145 \\
\hline \multicolumn{8}{|c|}{ Chr5: 52926659} \\
\hline \multirow[t]{5}{*}{$\mathrm{T}>\mathrm{A}$} & CK & 0.549554 & 0.450446 & 1.819657 & 0.348995 & 27.39184 & $<0.0001$ \\
\hline & JY & 0.536675 & 0.463325 & 1.863324 & 0.35599 & 0.739498 & 0.389822 \\
\hline & SM & 0.51020 & 0.489796 & 1.96 & 0.369846 & 16.67361 & $<0.0001$ \\
\hline & $\mathrm{TC}$ & 0.502551 & 0.497449 & 1.989848 & 0.373721 & 18.57106 & $<0.0001$ \\
\hline & Total & 0.53491 & 0.465086 & 1.86946 & 0.356934 & 2.282563 & 0.130836 \\
\hline \multicolumn{8}{|c|}{ Chr5: 52926664} \\
\hline \multirow[t]{5}{*}{$\mathrm{T}>\mathrm{C}$} & CK & 0.520748 & 0.479252 & 1.920316 & 0.364411 & 6.357222 & 0.01169 \\
\hline & JY & 0.712665 & 0.287335 & 1.403183 & 0.246054 & 2.556213 & 0.109861 \\
\hline & SM & 0.610162 & 0.389838 & 1.638908 & 0.313851 & 1.292205 & 0.255642 \\
\hline & $\mathrm{TC}$ & 0.518141 & 0.481859 & 1.929978 & 0.365765 & 9.771903 & 0.001772 \\
\hline & Total & 0.569586 & 0.430414 & 1.755662 & 0.337786 & 0.119836 & 0.729212 \\
\hline \multicolumn{8}{|c|}{ Chr3: 7519896} \\
\hline \multirow[t]{5}{*}{$\mathrm{G}>\mathrm{A}$} & CK & 0.625 & 0.375 & 1.6 & 0.304688 & 6.828283 & 0.008973 \\
\hline & JY & 0.847222 & 0.152778 & 1.180328 & 0.141107 & 9.917355 & 0.001637 \\
\hline & SM & 0.583299 & 0.416701 & 1.714388 & 0.329881 & 3.450571 & 0.06323 \\
\hline & $\mathrm{TC}$ & 0.69161 & 0.30839 & 1.445902 & 0.260838 & 12.10089 & 0.000504 \\
\hline & Total & 0.504349 & 0.49565 & 1.9827 & 0.372816 & 54.30615 & $<0.0001$ \\
\hline \multicolumn{8}{|c|}{ Chr3: 7519918} \\
\hline \multirow{5}{*}{$\mathrm{G}>\mathrm{A}$} & $\mathrm{CK}$ & 0.53858 & 0.46142 & 1.856734 & 0.354966 & 5.683225 & 0.017128 \\
\hline & $J Y$ & 0.736328 & 0.263672 & 1.35809 & 0.22891 & 1.646091 & 0.199492 \\
\hline & SM & 0.546855 & 0.453145 & 1.828637 & 0.350475 & 3.825476 & 0.050479 \\
\hline & $\mathrm{TC}$ & 0.547902 & 0.452098 & 1.825142 & 0.349901 & 1.407982 & 0.235392 \\
\hline & Total & 0.501342 & 0.498658 & 1.994645 & 0.374328 & 6.193828 & 0.01282 \\
\hline \multicolumn{8}{|c|}{ Chr24: 5748832} \\
\hline \multirow[t]{5}{*}{$\mathrm{T}>\mathrm{C}$} & $\mathrm{CK}$ & 1 & 0 & 1 & 0 & & \\
\hline & JY & 0.670139 & 0.329861 & 1.492228 & 0.275457 & 0.005319 & 0.941863 \\
\hline & SM & 0.567472 & 0.432528 & 1.762202 & 0.338988 & 11.33585 & 0.00076 \\
\hline & $\mathrm{TC}$ & 0.613379 & 0.386621 & 1.630314 & 0.311883 & 42 & $<0.0001$ \\
\hline & Total & 0.611157 & 0.388843 & 1.63624 & 0.313243 & 84.62 & $<0.0001$ \\
\hline \multicolumn{8}{|c|}{ Chr24: 5748784} \\
\hline \multirow[t]{5}{*}{$A>G$} & CK & 0.654321 & 0.345679 & 1.528302 & 0.285932 & 4.408163 & 0.035767 \\
\hline & JY & 0.635634 & 0.364366 & 1.573233 & 0.297985 & 11.3098 & 0.000771 \\
\hline & SM & 0.510204 & 0.489796 & 1.96 & 0.369846 & 5.444444 & 0.019631 \\
\hline & $\mathrm{TC}$ & 0.637188 & 0.362812 & 1.569395 & 0.296996 & 22.84406 & $<0.0001$ \\
\hline & Total & 0.500483 & 0.49951 & 1.998069 & 0.374758 & 59.32124 & $<0.0001$ \\
\hline
\end{tabular}

$\mathrm{CK}=$ Caoke chicken; JY = Jiuyuan black chicken; $\mathrm{SM}=$ Sichuan mountain chicken; $\mathrm{TC}=\mathrm{Tibetan}$ chicken; $H_{\mathrm{O}}=$ observed heterozygosity; $H_{\mathrm{E}}=$ expected heterozygosity; $N_{\mathrm{A}}=$ number of alleles; PIC = polymorphism information content; $\chi^{2}$ and its associated $\mathrm{P}$ value indicate significant deviations from Hardy-Weinberg equilibrium. 
Table 4. Genotype and allele frequencies of the seven SNP loci in four Chinese indigenous chicken breeds.

\begin{tabular}{|c|c|c|c|c|c|c|c|c|c|}
\hline \multirow{2}{*}{$\frac{\text { SNP }}{\text { Chr5: } 52922938}$} & \multirow[t]{2}{*}{ Genotype } & \multicolumn{2}{|c|}{ CK number/frequency } & \multicolumn{2}{|c|}{ JY number/frequency } & \multicolumn{2}{|c|}{ SM number/frequency } & \multirow[t]{2}{*}{$\mathrm{TC}$} & \multirow[t]{2}{*}{ number/frequency } \\
\hline & & & & & & & & & \\
\hline \multirow[t]{5}{*}{$A>G$} & AA & 46 & 0.851852 & 0 & 0 & 0 & 0 & 32 & 0.761905 \\
\hline & $\mathrm{AG}$ & 7 & 0.12963 & 48 & 1 & 0 & 0 & 0 & 0 \\
\hline & GG & 1 & 0.018519 & 0 & 0 & 49 & 1 & 10 & 0.238095 \\
\hline & A & & 0.916667 & & 0.5 & & 0 & & 0.761905 \\
\hline & G & & 0.083333 & & 0.5 & & 1 & & 0.238095 \\
\hline \multicolumn{10}{|l|}{ Chr5: 52926659} \\
\hline \multirow[t]{5}{*}{$\mathrm{A}>\mathrm{T}$} & AA & 32 & 0.592593 & 18 & 0.375 & 23 & 0.469388 & 16 & 0.380952 \\
\hline & AT & 7 & 0.12963 & 25 & 0.520833 & 10 & 0.204082 & 7 & 0.166667 \\
\hline & TT & 15 & 0.277778 & 5 & 0.104167 & 16 & 0.326531 & 19 & 0.452381 \\
\hline & A & & 0.657407 & & 0.635417 & & 0.571429 & & 0.464286 \\
\hline & $\mathrm{T}$ & & 0.342593 & & 0.364583 & & 0.428571 & & 0.535714 \\
\hline \multicolumn{10}{|l|}{ Chr5: 52926664} \\
\hline \multirow[t]{5}{*}{$\mathrm{C}>\mathrm{T}$} & $\mathrm{CC}$ & 24 & 0.444444 & 30 & 0.625 & 28 & 0.571429 & 10 & 0.238095 \\
\hline & $\mathrm{CT}$ & 17 & 0.314815 & 18 & 0.375 & 16 & 0.326531 & 30 & 0.714286 \\
\hline & TT & 13 & 0.240741 & 0 & 0 & 5 & 0.102041 & 2 & 0.047619 \\
\hline & $\mathrm{C}$ & & 0.601852 & & 0.8125 & & 0.734694 & & 0.595238 \\
\hline & $\mathrm{T}$ & & 0.398148 & & 0.1875 & & 0.265306 & & 0.404762 \\
\hline \multicolumn{10}{|l|}{ Chr3: 7319896} \\
\hline \multirow[t]{5}{*}{$\mathrm{G}>\mathrm{A}$} & GG & 28 & 0.518519 & 42 & 0.875 & 7 & 0.142857 & 5 & 0.119048 \\
\hline & GA & 10 & 0.185185 & 4 & 0.083333 & 15 & 0.306122 & 6 & 0.142857 \\
\hline & AA & 16 & 0.296296 & 2 & 0.041667 & 27 & 0.55102 & 31 & 0.738095 \\
\hline & G & & 0.611111 & & 0.916667 & & 0.295918 & & 0.190476 \\
\hline & $\mathrm{A}$ & & 0.388889 & & 0.083333 & & 0.704082 & & 0.809524 \\
\hline \multicolumn{10}{|l|}{ Chr3: 7519918} \\
\hline \multirow[t]{5}{*}{$\mathrm{G}>\mathrm{A}$} & AA & 18 & 0.333333 & 0 & 0 & 24 & 0.489796 & 20 & 0.47619 \\
\hline & AG & 33 & 0.611111 & 15 & 0.3125 & 16 & 0.326531 & 15 & 0.357143 \\
\hline & GG & 3 & 0.055556 & 33 & 0.6875 & 9 & 0.183673 & 7 & 0.166667 \\
\hline & A & & 0.638889 & & 0.15625 & & 0.653061 & & 0.654762 \\
\hline & $\mathrm{G}$ & & 0.361111 & & 0.84375 & & 0.346939 & & 0.345238 \\
\hline \multicolumn{10}{|l|}{ Chr24: 5748832} \\
\hline \multirow[t]{5}{*}{$\mathrm{T}>\mathrm{C}$} & TT & 0 & 0 & 2 & 0.041667 & 10 & 0.204082 & 31 & 0.738095 \\
\hline & $\mathrm{CT}$ & 0 & 0 & 16 & 0.333333 & 11 & 0.22449 & 0 & 0 \\
\hline & $\mathrm{CC}$ & 54 & 1 & 30 & 0.625 & 28 & 0.571429 & 11 & 0.261905 \\
\hline & $\mathrm{T}$ & & 0 & & 0.208333 & & 0.316327 & & 0.738095 \\
\hline & $\mathrm{C}$ & & 1 & & 0.791667 & & 0.683673 & & 0.261905 \\
\hline \multicolumn{10}{|l|}{ Chr24: 5748784} \\
\hline \multirow[t]{5}{*}{$\mathrm{A}>\mathrm{G}$} & AA & 0 & 0 & 32 & 0.666667 & 13 & 0.265306 & 30 & 0.714286 \\
\hline & $\mathrm{AG}$ & 24 & 0.444444 & 9 & 0.1875 & 16 & 0.326531 & 4 & 0.095238 \\
\hline & GG & 30 & 0.555556 & 7 & 0.145833 & 20 & 0.408163 & 8 & 0.190476 \\
\hline & $\mathrm{A}$ & & 0.222222 & & 0.760417 & & 0.428571 & & 0.761905 \\
\hline & $\mathrm{G}$ & & 0.777778 & & 0.239583 & & 0.571429 & & 0.238095 \\
\hline
\end{tabular}

$\mathrm{CK}=$ Caoke chicken; JY = Jiuyuan black chicken; $\mathrm{SM}=$ Sichuan mountain chicken; $\mathrm{TC}=$ Tibetan chicken.

The AG genotype of the Chr3:7519918G>A locus occurs mainly in Caoke chicken, the GG genotype occurs mainly in Jiuyuan black chicken, while the AA genotype occurs mainly in Sichuan mountain chicken and Tibetan chicken. The homozygous genotypes of the Chr24:5748832 $\mathrm{T}>\mathrm{C}$ and $\mathrm{Chr} 24: 5748784 \mathrm{~A}>\mathrm{G}$ loci are observed in all four breeds (Table 4).

\section{SNP locus phylogenetic analysis}

Phylogenetic analysis of the 7 SNPs in the four chicken breeds was undertaken and the results show that the internal genetic distance was 0.0122 in Caoke chicken, 0.0139 in Jiuyuan black chicken, 0.014 in Sichuan mountain chicken, and 0.0134 in Tibetan chicken. Tables 5, 6, and 7 detail the results of the genetic distance among the four chicken breeds. 
Table 5. Disparity and genetic distance within the four breeds of Chinese indigenous chicken.

\begin{tabular}{lcccc}
\hline Index & CK & JY & SM & TC \\
\hline Disparity index & 0.0042 & 0.0016 & 0.0018 & 0.0054 \\
Distance within groups & 0.0122 & 0.014 & 0.0139 & 0.0134 \\
\hline
\end{tabular}

$\mathrm{CK}=$ Caoke chicken; JY = Jiuyuan black chicken; $\mathrm{SM}=$ Sichuan mountain chicken; $\mathrm{TC}=$ Tibetan chicken.

Table 6. Mean genetic distance between Chinese indigenous chicken breeds and layer and broiler chicken.

\begin{tabular}{|c|c|c|c|c|c|c|}
\hline Breed & Layer & Broiler & CK & Gallus & SM & JY \\
\hline Layer & - & & & & & \\
\hline Broiler & 0.0416 & - & & & & \\
\hline CK & 0.0307 & 0.0104 & - & & & \\
\hline Gallus & 0.0234 & 0.0174 & 0.0225 & - & & \\
\hline SM & 0.0145 & 0.0264 & 0.0196 & 0.0245 & - & \\
\hline JY & 0.0163 & 0.0247 & 0.0191 & 0.0243 & 0.0153 & - \\
\hline $\mathrm{TC}$ & 0.0158 & 0.0252 & 0.0230 & 0.0125 & 0.0194 & 0.0180 \\
\hline
\end{tabular}

\begin{tabular}{|c|c|c|c|c|c|c|}
\hline Breed & Broiler & Layer & CK & Gallus & SM & JY \\
\hline Broiler & - & & & & & \\
\hline Layer & 0.0057 & - & & & & \\
\hline CK & 0.0173 & 0.0110 & - & & & \\
\hline Gallus & 0.0171 & 0.0057 & 0.0210 & - & & \\
\hline SM & 0.0105 & 0.0111 & 0.0152 & 0.0246 & - & \\
\hline$J Y$ & 0.0136 & 0.0135 & 0.0138 & 0.0293 & 0.0117 & - \\
\hline $\mathrm{TC}$ & 0.0144 & 0.0105 & 0.0171 & 0.0171 & 0.0169 & 0.0176 \\
\hline
\end{tabular}

\section{DISCUSSION}

Research by Li et al. (2012) suggested that genes related to chicken resistance traits are located on chromosome 16, and at the same time, they also located both the egg laying performance and growth performance genes. In our study, the objective genes were $L H C G R$, $E S R 2$, and $D R D 2$, located on chromosomes 3, 5, and 24, respectively, and our results agree with those of Li et al. (2013). Several studies have revealed that these three genes are related to reproduction traits in animals and humans (Greene et al., 1986; Maxwell et al., 1987; Duan et al., 2003; Kossack et al., 2008; Xu et al., 2010; Yang et al., 2012; Yu et al., 2012). As the results of the objective gene screening show, the loci are mainly located in non-coding regions, where proteins are not encoded, indicating that the mutation site does not change the gene function through changes to the protein. It may be that the mutation site plays a role by reducing the coding region mutation, as demonstrated in previous research (Akey et al., 2002; Matukumalli et al., 2006; Groenen et al., 2011). The function of the mutation site requires further investigation.

Locus polymorphism, a form of genetic polymorphism, is the result of both natural and manual selection; however, natural selection plays the main role and three levels of poly- 
morphism are assumed: low (PIC $<0.25)$, moderate $(0.25<$ PIC $<0.5)$ and high (PIC $>0.5)$ (Akey et al., 2002). Based on the results of the analyses conducted in the current study, we found that loci specific to layer chicken exhibited low polymorphism in the Caoke chicken breed, indicating that Caoke chicken has low levels of egg performance traits under natural selection. While the Jiuyuan black chicken breed exhibited moderate polymorphism for loci specific to layer chicken. In order to improve genetic progress, manual selection should be consistent with natural selection, thus, when we determine the breeding direction it should agree with natural selection. As the results show, some loci deviated from Hardy-Weinberg equilibrium, especially in the Tibetan chicken breed. Such deviations from Hardy-Weinberg equilibrium can be a result of sampling error caused by genetic drift. Deviations from HardyWeinberg equilibrium for loci in the Tibetan chicken breed may be a consequence of their living environment, where the altitude is $2500 \mathrm{~m}$. Further sampling of this population would help to eliminate the sampling error caused by genetic drift in future research. At the same time, we infer that the Tibetan chicken breed has diverged as a subspecies due to its geographical distribution and living environment.

Based on the genetic distance analysis, the Caoke chicken breed has the smallest genetic distance and largest coefficient of variation compared to the other three breeds, which indicates that Caoke chicken has high consistency and may have been introduced to the other breed's blood. The genetic distance between each breed indicates that genetic distance was greatest between layer and broiler chicken breeds. Of the experimental groups, the genetic distance between the Caoke chicken breed and broiler chicken was smaller than between the Caoke chicken breed and layer chicken, while the genetic distance between the Jiuyuan black chicken breed and broiler chicken was greater than between the Jiuyuan black chicken breed and layer chicken. The Sichuan mountain chicken and Tibetan chicken breeds exhibited no significant differences.

\section{CONCLUSION}

Variation observed in the $\mathrm{Chr} 24: 5748784 \mathrm{~A}>\mathrm{G}, \mathrm{Chr} 24: 5748832 \mathrm{~T}>\mathrm{C}$, and $\mathrm{Chr} 5: 529229$

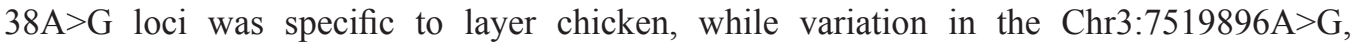
Chr3:7519918G $>$ A, Chr5:52926659T $>$ A, and Chr5:52926664T $>$ C loci was specific to broiler chicken.

Following analysis of the data, we suggest the Caoke chicken breed could be suitable for development in meat production, while the Jiuyuan chicken breed could be suitable for development in egg production. As the Sichuan mountain chicken and the Tibetan chicken exhibited large polymorphisms, these breeds could be improved by changing their living environment.

\section{Conflicts of interest}

The authors declare no conflict of interest.

\section{ACKNOWLEDGMENTS}

Research supported by the Program for the Key Project from the Education Department of Sichuan Province (\#14ZA0021), the Project of the Application Basis Project from the 
Science and Technology Department of Sichuan Province (\#2014JY0163), and the Project of National Natural Science Foundation of China (\#31402068), the project of Twelfth Five-Year Province Crops and Livestock and Poultry Breeding Research (\#2011NZ0099-7).

\section{REFERENCES}

Akey JM, Zhang G, Zhang K, Jin L, et al. (2002). Interrogating a high-density SNP map for signatures of natural selection. Genome Res. 12: 1805-1814.

Duan J, Wainwright MS, Comeron JM, Saitou N, et al. (2003). Synonymous mutations in the human dopamine receptor D2 (DRD2) affect mRNA stability and synthesis of the receptor. Hum. Mol. Genet. 12: 205-216.

Greene GL, Gilna P, Waterfield M, Baker A, et al. (1986). Sequence and expression of human estrogen receptor complementary DNA. Science 231: 1150-1154.

Groenen MA, Megens HJ, Zare Y, Warren WC, et al. (2011). The development and characterization of a 60K SNP chip for chicken. BMC Genomics 12: 274.

Gu X, Feng C, Ma L, Song C, et al. (2011). Genome-wide association study of body weight in chicken F2 resource population. PLoS One 6: e21872.

Kalinowski ST, Taper M L and Marshall TC (2007). Revising how the computer program CERVUS accommodates genotyping error increases success in paternity assignment. Mol. Ecol. 16: 1099-1106.

Kossack N, Simoni M, Richter-Unruh, A, Themmen AP, et al. (2008). Mutations in a novel, cryptic exon of the luteinizing hormone/chorionic gonadotropin receptor gene cause male pseudohermaphroditism. PloS Med. 5: e88.

Li DF, Liu WB, Liu JF, Yi GQ, et al. (2012). Whole-genome scan for signatures of recent selection reveals loci associated with important traits in White Leghorn chickens. Poult. Sci. 91: 1804-1812.

Li DF, Lian L, Qu LJ, Chen YM, et al. (2013). A genome-wide SNP scan reveals two loci associated with the chicken resistance to Marek's disease. Anim. Genet. 44: 217-222.

Matukumalli LK, Grefenstette JJ, Hyten DL, Choi IY, et al. (2006). Application of machine learning in SNP discovery. BMC Bioinformatics 7: 4 .

Maxwell BL, Mcdonnell DP, Conneely OM, Schulz TZ, et al. (1987). Structural organization and regulation of the chicken estrogen receptor. Mol. Endocrinol. 1: 25-35.

Peakall R and Smouse PE (2012). GenAlEx 6.5: genetic analysis in Excel. Population genetic software for teaching and research--an update. Bioinformatics 28: 2537-2539.

Reich DE, Gabriel SB and Altshuler D (2003). Quality and completeness of SNP databases. Nat. Genet. 33: 457-458.

Rubin CJ, Zody MC, Eriksson J, Measows JR, et al. (2010). Whole-genome resequencing reveals loci under selection during chicken domestication. Nature 464: 587-591.

Useche FJ, Gao G, Harafey M and Rafalski A (2001). High-throughput identification, database storage and analysis of SNPs in EST sequences. Genome Inform. 12: 194-203.

van Kaam JB, Groenen MA, Bovenhuis H, Veenendaal A, et al. (1999). Whole genome scan in chickens for quantitative trait loci affecting growth and feed efficiency. Poult. Sci. 78: 15-23.

Xie L, Luo C, Zhang C, Zhang R, et al. (2012). Genome-wide association study identified a narrow chromosome 1 region associated with chicken growth traits. PLoS One 7: e30910.

Xu HP, Shen X, Zhou M, Luo CL, et al. (2010). The dopamine D2 receptor gene polymorphisms associated with chicken broodiness. Poult. Sci. 89: 428-438.

Yang WC, Tang KQ, Li SJ, Chao LM, et al. (2012). Polymorphisms of the bovine luteinizing hormone/choriogonadotropin receptor (LHCGR) gene and its association with superovulation traits. Mol. Biol. Rep. 39: 2481-2487.

Yu Y, Pang Y, Zhao H, Xu X, et al. (2012). Association of a missense mutation in the luteinizing hormone/ choriogonadotropin receptor gene (LHCGR) with superovulation traits in Chinese Holstein heifers. J. Anim. Sci. Biotechnol. 3: 35. 